\title{
Effect of Microbubbles on Ozonized Water for Photoresist Removal
}

\author{
Masayoshi Takahashi, Hideo Horibe*, Kouhei Matsuura* and Katsumi Tatera** \\ National Institute of Advanced Industrial Science and Technology (AIST), \\ 16-1 Onogawa, Tsukuba, Ibaraki 305-8569, Japan \\ * Osaka City University, \\ 3-3-138 Sugimoto Sumiyoshi-ku, Osaka-shi, 558-8585, Japan \\ **Opt Creation, Inc. \\ Kasho Bldg.4F, 3014-2, Shinohara-cho, Kohoku, Yokohama, Kanagawa, \\ 222-0026, Japan \\ m.taka@aist.go.jp
}

\begin{abstract}
The existence of microbubbles in ozonized water has been shown to significantly enhance the photoresist removal rate due to an elevated dissolved ozone concentration and a direct effect of the microbubbles relating to the radical generation. Additionally, the ozone microbubble solution was able to effectively remove a high-dose ion-implanted photoresist, which is extremely resistant to removal by ozonized water and other wet chemicals because of its amorphous carbon-like layer, or "crust". Electron spin resonance experiments were also performed without the influence of serious metal contamination and indicated the presence of hydroxyl radicals, which are thought to be formed by interaction of ozone with hydroxide ions adsorbed at the gas-water interface upon collapse of the microbubbles.
\end{abstract}

Keywords: Ozone microbubbles, Photoresist removal, Hydroxyl radicals

\section{Introduction}

Microbubbles are tiny bubbles with diameters of less than $50 \mu \mathrm{m}$. When generated in water, the bubbles decrease in size and eventually disappear under water because of their long stagnation and excellent gas dissolution ability (Fig.1) [1]. The collapse of these bubbles in water has been shown to lead to generation of activated oxidizers such as hydroxyl radicals. This is thought to arise from the extinction of the gas-water interface, which might trigger dispersion of the elevated chemical potential accumulated at the interface as adsorbed ions [2-4]. Similarly, when microbubbles of ozone are generated in water, the dispersion of the chemical potential arising from the extinction of the gas-water interface leads to the decomposition of ozone and the generation of large quantities of hydroxyl radicals, which may provide a new type of advanced oxidation process (AOP) [5]. Such properties suggest the use of microbubbles as a new method of wastewater treatment.

In the production of semiconductor devices, surface contamination of the silicon wafers causes serious problems. As a result, large amounts of chemicals, such as $\mathrm{H}_{2} \mathrm{SO}_{4}, \mathrm{H}_{2} \mathrm{O}_{2}, \mathrm{HCl}, \mathrm{NH}_{4} \mathrm{OH}$, and amine solvents, are required in wet cleaning processes $[6,7]$. For safety and environmental reasons, the use of ozonized water is attracting attention as a viable method for removing organic contaminants, which has been conventionally removed by a high temperature sulfuric acid - hydrogen peroxide mixture (SPM) $[8,9]$. In terms of applications to semiconductor manufacturing, ozonized water cleaning systems are strongly desired as they provide enhanced removal rates for organic contaminants. In addition, the challenge to the amorphous carbon like damage layer created by ion-implant doses greater than $5 \mathrm{E} 14 / \mathrm{cm}^{2}$ is significantly important to introduce the ozone cleaning method in the semiconductor manufacturing processes $[10,11]$.

In this paper, we investigate the effects of microbubbles on ozonized water for the removal of photoresists. A comparison is made between treatments with and without microbubbles. We also discuss the physicochemical mechanism of ozone microbubbles in 


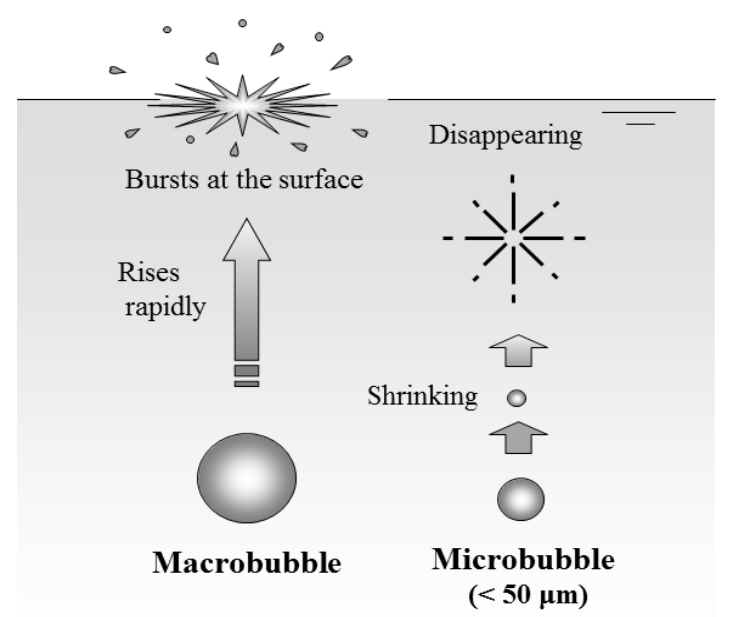

Fig.1. Microbubble behavior. The ordinary macrobubbles rose rapidly and burst at the surface of the water. By contrast, the microbubbles decreased in size and disappeared under the water.

enhancing the removal rate, taking into account the electron spin resonance (ESR) results, and describe the treatment of a high-dose ion-implanted photoresist.

\section{Ozone microbubble generation system for semiconductor production processes}

Fig. 2 shows a photograph of the microbubble generation system used in the experiments. For practical application in semiconductor manufacturing, all parts of the system in contact with water are made of metal-free Teflon materials. A diaphragm pump realizes low pulsation discharge intakes of water and gas through its intake line, and discharges them through a dissolution tank in which the gas is effectively dissolved in water under a pressure of approximately 0.4 MPa. The water containing the dissolved gas is then released by a dispersing nozzle, and the resulting pressure decreased to ambient conditions causes supersaturation of the dissolved gas. Bubble nuclei generated by turbulent flow at the nozzle grow rapidly into microbubbles under the supersaturated condition. Fig. 3 shows the bubble size distribution of generated microbubbles in distilled water measured by a particle-counting spectrometer for liquids (LiQuilaz-E20; Particle Measuring Systems Inc.) using a light-obscuration method. The microbubble solution, milky in appearance, exhibits two peaks, a sharp peak around $15 \mu \mathrm{m}$ in diameter and a broader peak around 40 $\mu \mathrm{m}$.

\section{Photoresist removal by ozone microbubbles}

The performance of the ozone microbubble solution

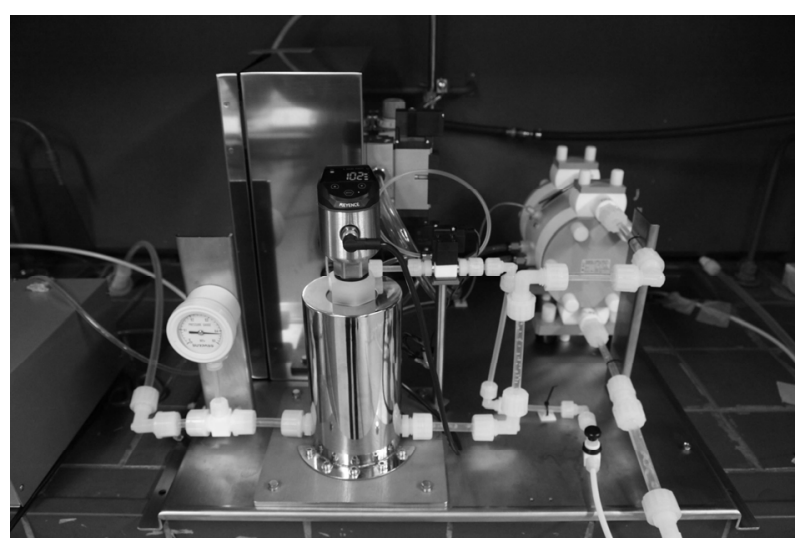

Fig.2 Photograph of the microbubble generation system for semiconductor cleaning

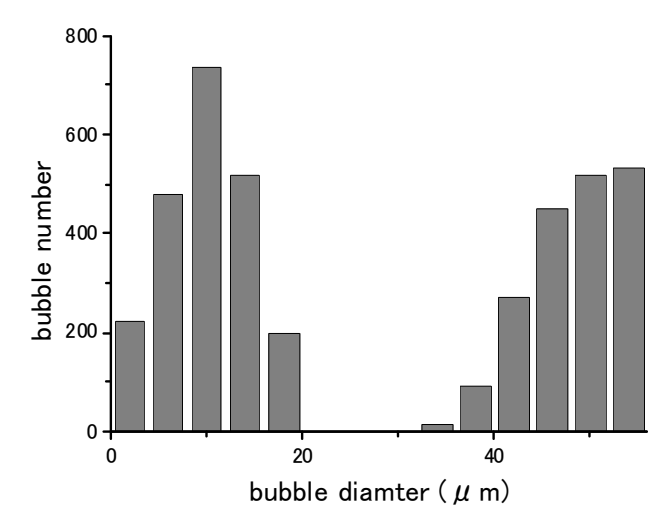

Fig. 3. Size distribution of the microbubbles generated by the apparatus.

in photoresist removal was investigated by measuring the change in thickness of the photoresist residue. A six-inch silicon wafer coated with unexposed high-contrast i-line positive photoresist (THMR-iP3300, TOKYO OHKA KOGYO Co. Inc.) was diced into test pieces of 30 $\mathrm{mm} \times 30 \mathrm{~mm}$. The tests were conducted by pouring the microbubble solution onto the diced wafers. In this test, the flow rate of the microbubble solution was approximately $8 \mathrm{~L} / \mathrm{min}$, which was sufficient to cover the entire surface of the diced wafer, ensuring uniform removal of the photoresist. The average thickness of nine distinct measurement points on the wafer was obtained from measurements taken every $30 \mathrm{~s}$ for 2 min using a thin film analyzer (F20, Filmetrics Inc.). The dissolved ozone concentration was approximately $15 \mathrm{mg} / \mathrm{L}$ and the water temperature was approximately $30^{\circ} \mathrm{C}$ throughout the test.

Fig.4 shows the thickness of the photoresist that was removed during the treatment as indicated by the difference in thickness from the original value. The 


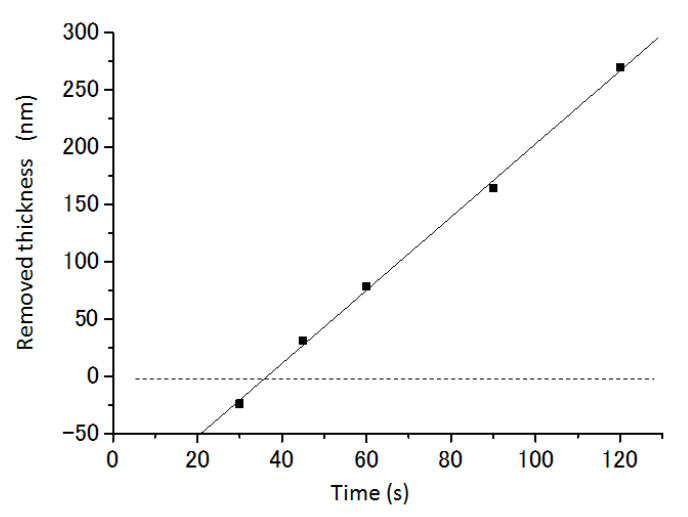

Fig. 4. Thickness of the photoresist that was removed by the ozone microbubble treatment with the dissolved ozone concentration of $15 \mathrm{mg} / \mathrm{L}$.

negative value at $30 \mathrm{~s}$ might be attributed to swelling of the photoresist layer due to action of the ozone microbubbles, and during this initial stage, it was also found that the surface changed from hydrophobic to hydrophilic. After this initial stage, there is an almost linear decrease in the thickness of the photoresist over time.

When applying ozonized water in semiconductor cleaning, an important factor to consider is how the rate of photoresist removal could be increased. A determining factor in this process has been shown to be the ozone concentration. Thus, we investigated the effect of ozone concentration of the source gas supplied for microbubble generation on the rate of photoresist removal. The tests were conducted under the same conditions as shown in Figure 4 with the exception that the ozone gas concentration was varied from 30 to $150 \mathrm{~g} / \mathrm{m}^{3}$. The removal rate of photoresist was evaluated by measuring the thickness of the photoresist over time by the thin film analyzer. It has been confirmed that there is a linear relationship between the ozone gas concentration and the photoresist removal rate. In terms of the photoresist removal rate, the increase in ozone gas concentration has the same effect in the microbubble treatment as that in the ordinary ozone treatment. And to evaluate the effect of microbubbles on photoresist removal we also conducted further tests to compare the removal rate obtained using ozonized water and an ozone microbubble solution, and it has been demonstrated that the presence of microbubbles in the ozonized water enhances the removal rate by more than $30 \%$ in comparison to the ozonized water without microbubbles.

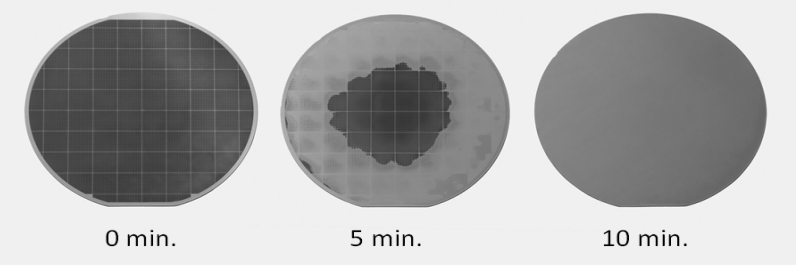

Fig. 5. Removal of high-dose ion-implanted photoresist by ozone microbubbles without any pretreatment.

\section{Removal of high-dose ion-implanted photoresist by ozone microbubble water}

The previous tests demonstrated enhancement in the photoresist removal rate by treatment with ozone microbubbles. However, in terms of practical application in semiconductor manufacturing, it would also be useful to remove photoresists damaged during manufacturing processes. One of the most challenging targets for the industry in terms of cleaning is the treatment of high-dose ion-implanted photoresists. Cleaning such photoresists is a considerable challenge due to an amorphous carbon-like damage layer (crust) caused by ion-implantation at doses higher than $5 \mathrm{E} 14 / \mathrm{cm}^{2}$, and conventionally a two-step process is used for photoresist removal; low-pressure plasma ashing in a single-wafer tool followed by SPM-based wet removal in a batch immersion tool. For advanced semiconductor fabrication processes, single-wafer tools are preferable as they prevent cross-contamination. Therefore, we then conducted tests on implementation of the ozone microbubble generation system to a conventional single-wafer spin cleaning tool.

Eight-inch silicon wafers, spin-coated with a 0.129 $\mu \mathrm{m}$ i-line positive photoresist (TDMR AR87LB-18G, TOKYO OHKA KOGYO Co. Inc.) and patterned by an exposure tool, were used as the test samples after phosphorus-ion implantation at a dose of $1 \mathrm{E} 15 / \mathrm{cm}^{2}$. The ion energy was $60 \mathrm{keV}$. The tests were conducted by pouring the ozone microbubble solution onto the wafers, which were rotating at a spin rate of $200 \mathrm{rpm}$. Ultrapure water was used with an electrical resistance of approximately $17 \mathrm{M} \Omega / \mathrm{cm}$. The dissolved ozone concentration was approximately $60 \mathrm{mg} / \mathrm{L}$ and the water temperature was approximately $22^{\circ} \mathrm{C}$ throughout the test. Figure 5 shows photographs of the wafer at 0,5 and 10 min after the start of treatment, which show that the 
high-dose ion-implanted photoresist was being removed from the outer edge to the center of the wafer. We also conducted the same test for an ozone solution without microbubbles. Significantly, the solution of dissolved ozone without microbubbles (also with a concentration of $60 \mathrm{mg} / \mathrm{L}$ ) did not remove any of the photoresist crust. Fig. 5 indicates another important factor to take into account when considering removal of the photoresist by the ozone microbubble solution. Because the lateral movement of the spinning wafer increases away from the central axis, the movement of the ozone microbubbles relative to the photoresist on the wafer also increases towards the outer edge of the wafer. The rapid relative movement of the water generates a turbulent flow beneath the surface. In fact, the observed trend of photoresist removal from the edge to the center may indicate that turbulent flow plays a significant role in removal of the photoresist. However, at present we do not have sufficient information to clarify the mechanism, and can only state that it may be a result of accelerated mass transfer of ozone to the surface of the wafer, or an effect of turbulent flow in collapsing of the microbubbles.

\section{Determination of hydroxyl radical generation by ESR measurement}

The above experiments demonstrate that the existence of microbubbles in ozonized water enhanced the photoresist removal rate for a backed photoresist and could effectively remove a high-dosed ion-implant photoresist. To clarify the mechanism by which the ozone microbubble solution acts on the photoresist, we performed ESR measurements by the spin-trap method. 5,5-dimethyl-1-pyrroline-N-oxide (DMPO) was used as the spin-trap reagent because it has been widely used to identify oxygen-centered radicals such as superoxide and the hydroxyl radical [12-16].

Ozonized water with or without microbubbles was tested by the ESR spin-trap method. Distilled water was used after the addition of ozone for the test. The microbubble generation system was used for the ozone microbubble solution, while the glass-bonded diffuser was used for the ozonized water without microbubbles. The electrical conductivity of the waters was approximately $1.0 \mu \mathrm{S} / \mathrm{cm}$, and the dissolved ozone concentration was approximately $8 \mathrm{mg} / \mathrm{L}$ in both cases. DMPO was mixed with the samples to a concentration of approximately $40 \mathrm{mM}$ under gentle stirring, and ESR spectra were measured at room temperature using an

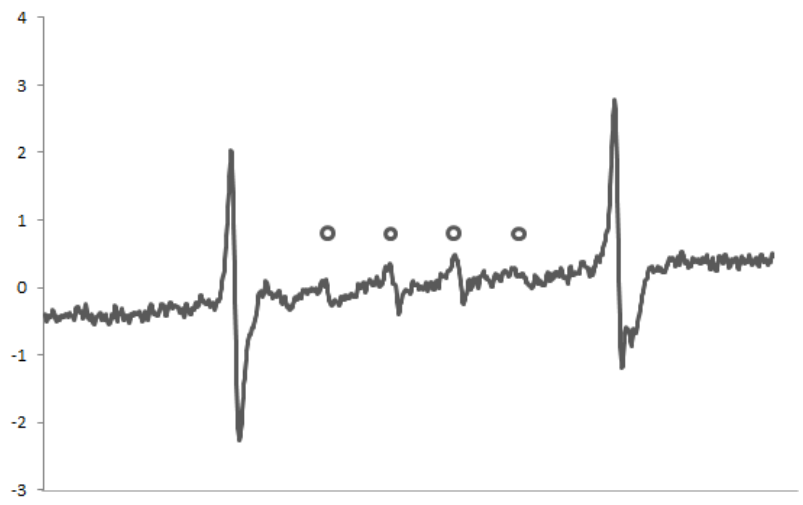

Fig.6. ESR spectrum of ozone microbubbles showing DMPO-OH. The four peaks in the mid-section (marked by circles) are signals of the DMPO-OH adduct, and the two peaks at each end are signals of $\mathrm{Mn}^{2+}$ (external reference).

ESR spectrometer (ESRX-10SA-v4, KEYCOM Co. Ltd.). The hyperfine splitting constant was calibrated using $\mathrm{Mn}^{2+}$ as an external standard. Figure 6 shows the ESR spectrum of the ozone microbubble solution, which contains four lines (hyperfine splitting constants of $A_{\mathrm{N}}=$ $\left.A_{\mathrm{H}}=14.9 \mathrm{G}\right)$, consistent with the spectra of DMPO-OH, indicating the formation of hydroxyl radicals $(\cdot \mathrm{OH})$. In the absence of microbubbles, we did not observe any spectral indications of hydroxyl radicals.

This finding is significant considering the photoresist removal because hydroxyl radicals have a much higher standard redox potential $(2.80 \mathrm{~V})$ than ozone $(2.07 \mathrm{~V})$, and exhibit immediate and non-selective reactivity with the majority of organic compounds. In terms of mechanism, the previous work has demonstrated that interfacial ions accumulated at the gas-water interface might play a role in the generation of free radicals during collapse of the microbubbles.

\section{Discussion}

Generation of free radicals is one of the most important features of microbubble treatment methods in applications such as disinfection and water treatment. The present work has revealed that microbubbles are very effective in photoresist removal because of the generation of hydroxyl radicals and an elevated concentration of dissolved ozone. Furthermore, the newly developed, metal-free microbubble generation system can produce microbubbles without serious contamination, enabling us to investigate the mechanism of transformation of ozone into hydroxyl radicals. 
Our experimental research into radical generation by collapsing microbubbles has revealed the following results $[1-5,17]$.

- The microbubbles decrease in size at an increasing rate and eventually disappear because of dissolution of the interior gas into solution.

- Zeta potential measurements reveal that the microbubbles are electrically charged owing to the adsorption of $\mathrm{H}^{+}$and $\mathrm{OH}^{-}$ions at the gas-water interface.

- In the collapse process, the increasing rate of shrinking of the gas-water interface leads to the accumulation of ions near the interface, resulting in a rapid increase in the absolute value of the zeta potential.

- The drastic environmental change caused by the extinction of the gas-water interface triggers radical generation via dispersion of the elevated chemical potential that has accumulated around the interface.

In pure water $\mathrm{H}+$ and $\mathrm{OH}-$ exclusively exit as ions, which are generated by the dissociation of water molecules. In a microbubble solution, these ions tend to be adsorbed at the gas-water interface, with $\mathrm{OH}$ - tending to be more effectively adsorbed at the interface than $\mathrm{H}+$, giving rise to a negative charge at the interface under a wide range of $\mathrm{pH}$ conditions. The negatively charged gas-water interfaces then attracts cations, that is, protons in this case, and generates an electrical double layer. Because the interior gases of the microbubbles are effectively dissolved into the surrounding water through the interface region, ozone molecules encounter accumulated hydroxide ions at the interface. This is almost the same situation for ozone as being dissolved in a high $\mathrm{pH}$ aqueous solution, and the radical chain that produces hydroxyl radicals is stimulated by the chemical reaction between ozone and hydroxide ions.

\section{Conclusion}

Ozonized water has attracted much attention as an environmental friendly cleaning method in semiconductor manufacturing, but its oxidative ability must be enhanced for practical application. Ozone microbubbles generated from a metal-free microbubble generator were investigated in the removal of photoresists from silicon wafers. It was found that the existence of microbubbles in ozonized water significantly enhances the photoresist removal rate due to the elevated dissolved ozone concentration and the collapse of the microbubbles. Moreover, the ozone microbubble solution was able to effectively remove a high-dose ion-implanted photoresist which is extremely resistant to removal by ozonized water and other wet chemicals. Electron spin resonance experiments revealed that the hydroxide ions adsorbed at the gas-water interface of the collapsing microbubbles may initiate the transformation of ozone into hydroxyl radicals, which play an important role in photoresist removal by the ozone microbubble treatment.

\section{Acknowledgments}

This study was partly supported by the Japan Science and Technology Agency (JST) and the Ministry of Economy, Trade and Industry (METI).

\section{References}

1. M. Takahashi, T. Kawamura, Y. Yamamoto, H. Ohnari, S. Himuro, H. Shakutui, J. Phys. Chem. B, 107 (2003) 2171.

2. M. Takahashi, K. Chiba, P. Li, J. Phys. Chem. B, 111 (2007) 1343.

3. P. Li, M. Takahashi, K. Chiba, Chemosphere, 75 (2009) 1371.

4. P. Li, M. Takahashi, K. Chiba, Chemosphere, 77 (2009) 1157.

5. M. Takahashi, K. Chiba, P. Li, J. Phys. Chem. B, 111 (2007) 11443.

6. M. N. Kawaguchi, J. S. Papanu, B. Su, M. Castle, A. Al-Bayati, J.Vac.Sci.Technol, B24 (2006) 657.

7. F. De Smedt, S. De Gendt. M. Heyns, C. J. Vinckier, Electrochem. Soc., 148 (2001) G487.

8. S.Noda, K. Kawase, H. Horibe, I.Oya, M. Kuzumoto, T. Kataoka, J. Electrochem. Soc., 152 (2005) G73.

9. H. Horibe, M. Yamamoto, T. Ichikawa, T. Kamimura, S. Tagawa, J. Photopolym. Sci. Technol. 20 (2007) 315.

10. P. M. Visintin, M. B. Korzenshi, T. H. Baum, J. Electrochem. Soc., 153 (2006) G591.

11. H. Utsumi, M. Hakuda, S. Shimbara, H. Nagaoka, Y. Chung, A. Hamada, Wat. Sci. Tech., 30 (1994) 91.

12. J. Lee, K. Park, S. Lim, J. Industrial and Engineering Chem., 14 (2008) 100.

13. Y. J. Kim, J. H. Lee, K. J. Seo, C. R. Yoon, E. S. Roh, J. K. Cho, T. Hattori, Solid State Phenomena, 269 (2009) 145. 
14. T. Hattori, Y. J. Kim, C. Yoon, J. K. Cho, IEEE Transsactions on Semiconductor Manufacturing, 22 (2009) 468.

15. D. S. Chou, G. Hsiao, M. Y. Shen, Y. J. Tsai, T. F. Chen, J. R. Sheu, Free Radic. Biol. Med., 39 (2005) 237.
16. L. H. Patterson, F. A. Taiwo, Biochem. Pharmacol,, 60 (2000) 1933.

17. M. Takahashi, J. Phys. Chem. B, 109 (2005) 21858. 